\title{
Neue mathematisch-statistische Anwendungen von Gesundheitsregistern
}

\author{
Klaus Krickeberg \\ Bielefeld, Deutschland
}

(Vortrag auf dem Gedenkkolloquium für Leopold Schmetterer)

Leopold Schmetterer war ein ungewöhnlich vielseitiger Mathematiker. In meinem Vortrag zu seinem 80. Geburtstag habe ich geschildert, wie er mir auch darin ein Vorbild gewesen ist und wie er mich beeinflusst hat. Seine medizinischen und pharmakologischen Interessen, wie sie sich zum Beispiel in seinen Untersuchungen zur stochastischen Approximation ausdrückten, haben dabei eine besondere Rolle gespielt. In dem damaligen Vortrag habe ich auch erzählt, wie verschiedene, zum Teil zufällige, Anlässe mich dazu gebracht haben, in dieser Richtung weiter zu gehen. Heute möchte ich über etwas berichten, was in jüngster Zeit dabei herausgekommen ist.

Im Titel steht etwas über Gesundheitsregister. Das kennt man aus Krankenhäusern, wo insbesondere die Gesamtheit der Krankenakten oder „Krankenblätter“ im logischmathematischen Sinne ein Register bildet, wenn es auch äußerlich nicht so aussieht, zumal die Krankenakten vor dem Gebrauch von Computern rasch von einer dicken Staubschicht überzogen wurden. Immerhin haben sie dazu gedient, viele Doktoren der Medizin zu produzieren. Es gibt die Tradition der so genannten statistischen Doktorarbeiten, die in der beschreibenden und meist wenig analysierenden Auswertung von Krankenakten bestehen. Der Doktorvater pflegte sie an Kandidaten zu vergeben, die er zu Besserem nicht fähig hielt, oder auch dann, wenn ihm kein anderes Thema einfiel. Im Prinzip können solche Arbeiten sehr aufschlussreich sein, wenn die Auswahl der Akten und ihre Analyse gemäß den mathematisch-statistischen Methoden der modernen Epidemiologie erfolgen, aber das ist selten der Fall.

In den reichen industrialisierten Ländern gibt es im Bereich der Gesundheit nur wenige Register und darauf aufbauende Informationssysteme, die über einzelne Krankenhäuser hinausreichen und das ganze Land überziehen. Dänemark hat zum Beispiel schon seit 1941 ein zentrales Krebsregister, aber selbst das findet man in den meisten entwickelten Ländern noch nicht. Register und Informationssysteme, die nicht nur solche speziellen Probleme wie Krebs betreffen, sondern das ganze Gesundheitswesen, existieren wohl nirgends.

Anders ist es in Entwicklungsländern. Das Gesundheitswesen in Entwicklungsländern hat meist eine sehr viel klarere und logischere Struktur als in entwickelten. Das mag erstaunen, aber es ist die natürliche Folge der Armut an Mitteln und an qualifiziertem Personal, die einen dazu zwingt, die Gesamtheit der Aktivitäten rationell zu organisieren und nichts zu verschwenden. Ein nach einheitlichen Gesichtspunkten strukturiertes staatliches Gesundheitswesen kann aber nur mit Hilfe eines Informationssystems funktionieren, das das Ganze ,,im Innersten zusammenhält““.

Ich habe hier einmal in einem anderen Vortrag ein solches Gesundheitsinformationssystem beschrieben, nämlich das vietnamesische. Das war vielleicht kein optimales Beispiel, denn dieses System ist recht schwerfällig und bürokratisch und enthält viel zu viele Informationen, die weder gebraucht noch ausgewertet werden. Das ist wohl eine Folge 
der vietnamesischen Geschichte: erst 1000 Jahre chinesische Herrschaft und Bürokratie, dann sehr viel später etwa 70 Jahre französische Bürokratie und schließlich noch eine reichliche Dosis sowjetischer, das hinterlässt Spuren.

Vor vier Monaten hatte ich Gelegenheit, mir das Gesundheitswesen und das dem zugrunde liegende Informationssystem von Burkina Faso anzusehen. In den Grundzügen ähnelt es dem vietnamesischen, weil sich eben die Gesundheitswesen der meisten Entwicklungsländer ähneln als Folge der schon erwähnten Notwendigkeit, rationell zu wirtschaften. Das Gesundheitsinformationssystem von Burkina Faso ist aber viel einfacher, durchsichtiger, und zweckmäßiger als das vietnamesische.

Heute möchte ich mich nun mit dem im Titel genannten Teilaspekt von Gesundheitsinformationssystemen in Entwicklungsländern beschäftigen, der die Quelle aller Informationen bildet, nämlich mit den Registern. Unter ihnen will ich mich auf die wichtigsten beschränken, das sind die Register in den Gesundheitsstationen, und unter denen wieder auf das wichtigste, nämlich auf das Register der Konsultationen. Zuvor muss ich aber doch einige Dinge ins Gedächtnis zurückrufen.

In den meisten Entwicklungsländern wird die primäre Gesundheitspflege durch ein Netz von staatlichen Gesundheitsstationen vermittelt, die über das ganze Land verteilt sind. Typischerweise hat jede Gemeinde eine solche Station, die dann der Größenordnung nach 10.000 Leute versorgt. Sie enthält etwa 2, 3 oder 4 Räume, darunter einen für Konsultationen und einen mit ein paar Krankenbetten, vor allem für Entbindungen. Das Personal besteht aus 1 bis 2 Krankenpflegern oder Krankenschwestern und einer Hebamme; manchmal ist auch ein voll ausgebildeter Arzt dabei. Übrigens existiert so ein Netzwerk auch in Europa, nämlich in Griechenland und Zypern.

Das Netzwerk dieser Gemeindegesundheitsstationen wird im Prinzip zusammen mit den Krankenhäusern zentral vom Gesundheitsministerium verwaltet, über Zwischenstationen wie die Gesundheitsämter der Distrikte und Provinzen. Dazu braucht die Gesundheitsverwaltung Informationen, die die Form von statistischen Indikatoren haben. Zwei triviale Beispiele zur Illustration: erstens die Anzahl der Masernfälle in einer gegebenen Gemeinde und einem gegebenen Monat; das ist ein epidemiologischer Indikator; zweitens die Anzahl der Konsultationen, bei denen ein bestimmtes Medikament verabreicht worden ist; das wäre ein ökonomischer Indikator. Solche Indikatoren sollen also den höheren Instanzen regelmäßig mitgeteilt werden, und das geschieht durch Berichte, die z.B. monatlich von den Gesundheitsstationen an die Gesundheitsämter der Distrikte geschickt werden, von da in aggregierter Form weiter an die der Provinzen und schließlich von dort an das Ministerium.

Um diese Berichte zu schreiben, muss das Personal der Gesundheitsstationen die diversen Indikatoren erst einmal selbst berechnen, und das tut es natürlich mit Hilfe der Daten, die z.B. während der Konsultationen ,,registriert“, d.h. aufgeschrieben werden. Damit sind wir bei den Registern. Das Register der Konsultationen ist ein Buch mit einer Zeile pro Konsultation. Weiterhin hat es Spalten, und zwar entspricht jede Spalte einer Variablen, deren Werte für jede Konsultation registriert werden. Typische Variable wären: das Datum der Konsultation, der Name des Konsultanten, seine Adresse, Alter, Geschlecht, Beruf, Symptome, Diagnose, Behandlung.

Die Gesundheitsbehörden interessieren sich normalerweise nur für die Indikatoren und überlassen es den Krankenpflegern, wie sie diese aus den Daten der Register berech- 
nen und wie sie die Ergebnisse in die Formulare für die Berichte übertragen. Das ist einer der Hauptgründe dafür, dass viele Gesundheitsinformationssysteme, z.B. das vietnamesische, nicht gut funktionieren. In meinem damaligen Vortrag habe ich gezeigt, wie man Abhilfe schaffen kann, indem man die Register und die Formulare für die Berichte aufeinander abstimmt, so dass sie die Struktur der zu Grunde liegenden Arbeitsgänge wieder spiegeln, und wie man fehlerhafte oder fehlende Indikatoren mit modernen Methoden der mathematischen Statistik durch geschätzte Werte ersetzen kann.

Register sind aber nicht nur zum Berechnen und zur Berichterstattung von Indikatoren da, obwohl die Bürokraten der Gesundheitsverwaltungen das meist so sehen. Sie existieren zuerst, und vor allem, für die tägliche Arbeit der Gesundheitsstation selbst, genauso wie Krankenakten bei uns nicht in erster Linie zum Abfassen von Dissertationen dienen, sondern zur klinischen Arbeit. Eine Zeile im Register der Konsultationen entspricht ja im Grunde einer Krankenakte. Das Aufschreiben all dieser Daten für eine gegebene Konsultation hilft dem Krankenpfleger oder Arzt bei der Konsultation und der Behandlung des Falls.

Unser heutiges Thema ist nun das folgende: wozu können Register in Gesundheitsstationen von Entwicklungsländern außerdem noch dienen, also neben diesen beiden klassischen Funktionen der Unterstützung der täglichen klinischen Arbeit und der Berichterstattung über Indikatoren? Die Antwort lautet: zur Forschung, und zwar sowohl zur epidemiologischen Forschung als auch zu der über Gesundheitssysteme. Das ist natürlich zum größten Teil eine sehr angewandte Forschung über praktische Probleme. Aus Zeitmangel und weil ich das besser kenne will ich mich auf epidemiologische Probleme beschränken.

Epidemiologie ist ja, darüber habe ich einmal in dieser Akademie vorgetragen, das Studium von statistischen Relationen zwischen zwei bestimmten Typen von Variablen, nämlich den folgenden. Zum einen hat man eine Variable $X$, die das Auftreten oder Vorhandensein einer bestimmten Krankheit beschreibt und die man die Ergebnisvariable nennt. Zum anderen gibt es so genannte Vorhersagevariable, von denen man vermutet, dass sie $X$ irgendwie beeinflussen, z.B. als Risikofaktoren; die fassen wir zu einer vektorwertigen Variablen $Y$ zusammen. Definiert sind diese Variablen meistens für Personen oder auch für Konsultationen. Das beliebteste Beispiel ist wohl das folgende: $Y$ stellt Rauchgewohnheiten dar und $X$ das Auftreten von Lungenkrebs.

Eine normale epidemiologische Studie sieht so aus: man wählt Gruppen von Personen aus mit Hilfe eines bestimmten rigorosen Stichprobenplans, in den ein Zufallsmechanismus eingebaut ist. An diesen Personen misst man die Werte von $X$ und $Y$, und dann wertet man die so gewonnenen Daten mit geeigneten Methoden der mathematischen Statistik aus.

Je nach Art des Stichprobenplans unterscheidet man verschiedene Typen von epidemiologischen Studien. In einer Querschnittsstudie werden die Personen unabhängig von $X$ und $Y$ gewählt. In einer Fall-Kontroll-Studie hängt die Wahl von $X$ ab. Man nimmt nämlich eine Gruppe von Fällen, bei denen die Krankheit also vorliegt, und eine von Kontrollen, d.h. von nicht erkrankten Personen. In einer Kohortstudie hängt die Wahl dagegen von $Y$ ab. Man verschafft sich Gruppen von Personen, die sich durch verschiedene Werte von $Y$ unterscheiden, z.B. Raucher und Nichtraucher. Dann gibt es noch so genannte Hybridstudien, gemischte Verfahren, die mathematisch sehr kompliziert sein können. Über solche Studien habe ich hier einmal berichtet, als das Gebiet ganz neu war, und mir 
steht noch lebhaft vor Augen, wie Schmetterer sich dafür sehr interessierte. Inzwischen hat sich dieses Gebiet mächtig weiter entwickelt.

In jedem Fall erfordert eine korrekte epidemiologische Studie einen großen Aufwand. Zuerst müssen die teilnehmenden Personen definiert und rekrutiert werden. Das ist meist teuer und kompliziert, und deshalb hat man oft nicht genug Teilnehmer. Dann sind die Werte der betreffenden Variablen an diesen Personen zu messen, z.B. über Fragebögen; auch das ist nicht einfach. Es erscheint daher verlockend, die in den Registern schon vorhandenen Namen als Grundstock zu verwenden und die betreffenden Stichproben direkt daraus zu ziehen, was sehr einfach ist. Auch viele Daten liegen schon vor. Es gibt aber kaum systematische Untersuchungen darüber, was man eigentlich mit solchen registergestützten Studien machen oder auch nicht machen kann, genauso wie man sich anscheinend selten systematisch darüber Gedanken gemacht hat, was man alles aus Krankenakten herausholen kann. Wir wollen also dieses Problem angehen, können aber natürlich im Wesentlichen nur Beispiele behandeln.

Wie immer in der Statistik müssen wir zuerst darüber nachdenken, welche Bevölkerung wir eigentlich studieren. In den Registerstudien ist das die der Leute, die irgendwann zu einer Konsultation in die Gesundheitsstation kommen. Das sind zwar erheblich mehr als nur Krankenhauspatienten, und sie sind ziemlich repräsentativ für die Gesamtbevölkerung, aber rein logisch ist es doch etwas Anderes. Ich will daher zuerst einige Beispiele bringen, in denen man sich von vornherein in ganz natürlicher Weise gerade für diese Population interessiert. Das ist der Fall bei allen statistischen Problemen, die den Behandlungsgang eines Patienten betreffen, der zur Konsultation kam, von der Anamnese und den Symptomen über die Diagnose und Behandlung bis zum Ausgang.

Triviale Beispiele sind die Berechnung der epidemiologischen Indikatoren, von denen wir vorhin gesprochen haben, nämlich der Häufigkeiten der diversen diagnostizierten Krankheiten, die ,nach oben“ berichtet werden. Das gibt das so genannte Krankheitsspektrum der Gesundheitsstationen und findet Eingang in statistische Jahrbücher des Gesundheitswesens. Es handelt sich einfach um registergestützte Querschnittsstudien, die jeden Monat wiederholt werden.

Interessanter wird es, wenn wir uns fragen, ob die dabei verwendeten Diagnosen auch stimmen. Das Problem existiert natürlich auch bei uns, hat aber in den Gesundheitsstationen von Entwicklungsländern eine viel größere Bedeutung, weil es an technischen Mitteln, an Laboratorien, und manchmal auch an gut ausgebildetem Personal fehlt. Nun sind Diagnoseverfahren de facto meist ziemlich standardisiert, nämlich festgelegt durch die Gewohnheiten der Gesundheitspfleger und Ärzte und manchmal sogar durch explizite Entscheidungsregeln. In anderen Worten, die Diagnose ist eine mehr oder weniger wohl bestimmte Funktion $d(s)$ wobei $s$ gewisse Symptome darstellt; die Werte von $d$ sind ,+"“ und ,--“. An dieser Entscheidungsfunktion $d$ interessieren uns vier Charakteristiken, nämlich ihre Sensitivität, d.h. die Wahrscheinlichkeit einer positiven Diagnose, wenn der Konsultant wirklich an der betreffenden Krankheit leidet; ihre Spezifizität, d.h. die Wahrscheinlichkeit einer negativen Diagnose, wenn sie bei ihm nicht vorliegt; und ihre beiden Vorhersagewerte, d.h. die Wahrscheinlichkeit für das Vorliegen bzw. Nichtvorliegen der Krankheit, wenn die Diagnose positiv bzw. negativ war.

In den Gesundheitsstationen von Entwicklungsländern registriert man fast nur rein klinische Symptome und leitet daraus die Diagnose ab. Ein Beispiel, das ich in Kambodscha 
in Gebieten, in denen Malaria endemisch war, gesehen habe: bei jedem Patienten mit Fieber und Kopfschmerzen lautet die Diagnose sofort auf Malaria. Ist das vernünftig? In Wien wäre es das sicher nicht. Um diese Frage unter den kambodschanischen Bedingungen zu beantworten, muss man die vier Charakteristiken dieser Diagnosefunktion schätzen. Das kann man aber im Falle der Malaria und mancher anderer chronischer Krankheiten mit einer allein auf das Register der Konsultationen gestützten Querschnittsstudie machen, weil nämlich das tatsächliche Vorliegen oder Nichtvorliegen der Krankheit letzten Endes, wenn auch nicht sofort, bei den meisten Patienten bekannt wird und daher mit herangezogen werden kann.

Wie schätzt man die Charakteristiken aber, wenn das nicht so ist, d.h. wenn man die Patienten immer gleich wieder aus den Augen verliert wie bei vielen akuten Erkrankungen? Nehmen wir als Beispiel die Bakterienruhr (Shigellose). In einer normalen Gesundheitsstation hängt die Diagnose wie gesagt nur von klinischen Symptomen ab wie hier Häufigkeit und Beschaffenheit des Stuhls, Fieber und Kopfschmerzen. Ohne eine Untersuchung des Stuhls im Labor ist es jedoch nicht einfach, Bakterienruhr von Amöbenruhr zu unterscheiden. In diesem Fall kann man eine analoge Studie machen mit einer aus dem Konsultationsregister gezogenen Stichprobe, für die man jetzt aber die Mittel bereit stellt, eine solche Laboruntersuchung auszuführen.

Dieses Beispiel führt uns auf eine ganz allgemeine und bisher praktisch unbekannte Methode, nämlich auf die der teilweise auf Register gestützten Studien. Wir haben ja hier eine neue Variable zu den schon im Register vorhandenen hinzugefügt, sozusagen eine neue Spalte, nämlich das tatsächliche Vorliegen oder Nichtvorliegen von Bakterienruhr, wie es sich aus der Stuhluntersuchung im Labor ergibt. Wir tun das jedoch nur im Rahmen der Studie für die Patienten der aus dem Register gezogenen Stichprobe und nicht routinemäßig, weil das unter den Bedingungen eines Entwicklungslandes nicht möglich wäre. Eine teilweise registergestützte Studie ist also eine, in der zusätzlich zu den Variablen des Registers noch die Werte einiger anderer Variabler gemessen werden, aber eben nur innerhalb der Studie. Dazu muss man dann im Allgemeinen besondere Mittel einsetzen, die normalerweise nicht zur Verfügung stehen, wie etwa Laboruntersuchungen. Im Gegensatz zu einer normalen epidemiologischen Studie zieht man die Stichprobe aus dem Register und benutzt auch die ohnehin im Register vorhandenen Variablen.

Ich werde nachher noch andere Anwendungen dieser Methode erwähnen, möchte aber vorher etwas systematischer auf die Diagnosen eingehen. Warum interessieren wir uns eigentlich für ihre Charakteristiken und wollen sie schätzen?

Der erste Grund kommt aus der normalen, klassischen Gesundheitsstatistik. Ich sagte schon, dass die Gesundheitsbehörden die Häufigkeiten der verschiedenen Krankheiten kennen möchten, also das Krankheitsspektrum. Diese Indikatoren stammen aus den Berichten und stützen sich auf die in den Gesundheitsstationen gestellten Diagnosen, welche oft falsch sind. Wenn man aber die vier Charakteristiken dieser klinischen Diagnoseentscheidungsschemata ungefähr kennt, dann kann man damit die berichteten Häufigkeiten durch geschätzte Häufigkeiten ersetzen, die im allgemeinen und auf die Dauer den wirklichen Häufigkeiten sehr viel näher kommen. Dazu braucht man nur ganz elementare Bayessche Verfahren. So etwas liegt natürlich der Mentalität der Bürokraten im Gesundheitswesen völlig fern und wird daher so gut wie nie gemacht, wäre aber durchaus sinnvoll. Beispiele wären die erwähnten Malaria- und Shigellosediagnosen. 
Der zweite, damit verwandte Grund ist der folgende. In derselben teilweise registergestützten Querschnittsstudie kann man neben den Charakteristiken des tatsächlich verwendeten Diagnoseschemas auch die von anderen, hypothetischen Diagnoseentscheidungsverfahren schätzen, soweit sich diese ebenfalls nur auf leicht beobachtbare klinische Symptome stützen. Danach kann man diese verschiedenen diagnostischen Schemata miteinander vergleichen, um vielleicht ein besseres oder sogar in irgendeinem Sinne optimales Schema auszusuchen und dann in der Praxis anzuwenden.

Der dritte Grund für unser Interesse an der Diagnostik liegt in ihrer Rolle in dem vollständigen Ablauf der Konsultation einschließlich der Behandlung. In einem korrekten medizinischen Entscheidungsschema ist die Behandlung weit gehend durch die Diagnose determiniert, wenn auch andere Faktoren wie Kosten, Allgemeinzustand des Patienten usw. eine Rolle spielen mögen. In einer Gesundheitsstation eines Entwicklungslandes stützt man sich notwendigerweise auf die vorläufige, klinische Diagnose. Gehen wir zum Beispiel der Malaria in Kambodscha zurück. Dort bekommt eben jeder, der über Kopfschmerzen klagt und Fieber hat, sofort ein Malariamittel. Die Station besitzt nämlich kein Mikroskop, und bis das Resultat der mikroskopischen Untersuchung einer Blutprobe aus dem nächstgelegenen Distriktkrankenhaus zurückgekommen ist, kann viel Zeit vergehen. Bei uns gibt es so etwas natürlich auch, vor allem das Verabreichen von Antibiotika, insbesondere Breitbandantibiotika, ohne dass das Resultat einer Bakterienkultur bekannt wäre. Die Gründe sind aber andere. Ob nun diese therapeutische Strategie in Kambodscha sinnvoll oder gar optimal ist, das ließe sich wieder mit Hilfe einer allein auf dem Register der Konsultationen beruhenden Querschnittsstudie feststellen.

Eine interessante Variante dieses Problems liegt vor, wenn man therapeutische Strategien verschiedener Länder miteinander vergleichen will. Ein Beispiel einer gerade beginnenden Studie dieser Art betrifft die Behandlung der Echinokokkose. Das ist der Befall des Patienten mit dem so genannten Hundebandwurm, der je nach Art des Erregers zu großen Zysten oder zu vielen verzweigten Kleinzysten führt, besonders in der Leber, was die völlige Zerstörung der Leber nach sich ziehen kann. Die Echinokokkose ist u.a. in vielen Ländern des südlichen und östlichen Mittelmeerrandes verbreitet, von Marokko bis zur Türkei, und wird auch in manche europäische Länder verschleppt. Die wichtigste Motivation dieser Studie liegt darin, dass man in den diversen Gesundheitsstrukturen nach ziemlich undurchsichtigen Kriterien vier völlig verschiedene Behandlungsmethoden verwendet, und zwar auch bei ganz gleich gelagerten Fällen. Die erste Methode besteht in der operativen Entfernung der Zysten, die zweite in ihrer Drainage, die dritte im Verabreichen von Medikamenten, und die vierte im Abwarten und Beobachten. Daher erhebt sich die folgende Frage: welche dieser vier Strategien ist denn, nach Abwägen aller Vor- und Nachteile und in Abhängigkeit von der Ausgangslage, die beste? Nun werden die Patienten zwar meistens letzten Endes in Krankenhäusern behandelt, aber zuerst konsultieren sie im Allgemeinen eine Gesundheitsstation. Wir können daher die Wirksamkeit jeder dieser Therapien wieder durch Querschnittsstudien schätzen, die sich allein auf die Register der Gesundheitsstationen zusammen mit den Akten der beteiligten Krankenhäuser stützen.

Das wären also Beispiele von Querschnittstudien zur Auswertung vollständiger Behandlungsschemata, von der Diagnose bis zur Therapie. Ich habe das an erster Stelle und relativ ausführlich behandelt weil es, wie schon gesagt, die natürliche Population von Konsultationsregistern betrifft. Im öffentlichen Gesundheitswesen steht allerdings nicht 
die Behandlung im Vordergrund. Was uns zuerst interessiert, ist die Frage nach den Ursachen und der Entstehung von Krankheiten, also die Ätiologie, und an zweiter Stelle, damit zusammenhängend, die Prävention.

Sehen wir uns zunächst einmal ein Beispiel zur Prävention an. In Entwicklungsländern spielen ja immer noch Infektionskrankheiten die Hauptrolle, und eine der wichtigsten Methoden ihrer Prävention sind Impfungen. Um etwas Konkretes vor Augen zu haben, könnten wir an die Impfung gegen Masern denken, die normalerweise einmalig und zwar im ersten Lebensjahr erfolgt. An einem wohl definierten und systematisch ausgeführten Impfprogramm interessieren uns vor allem zwei Größen. Die erste ist die Wirksamkeit $h$ der Impfung, die mit dem Ablauf des Programms nichts zu tun hat und mit Hilfe eines Gedankenexperiments definiert wird. Vereinfacht gesagt ist sie der Bruchteil der durch die Impfung vermiedenen Masernfälle unter den Fällen, die es ohne Impfung gegeben hätte. Die zweite Größe heißt der Durchimpfungsgrad g, der den wichtigsten Aspekt des Ablaufs des Programms beschreibt, nämlich den Bruchteil aller Kinder, die vor dem Ende ihres ersten Lebensjahres gegen Masern geimpft worden sind.

Den Durchimpfungsgrad kann man im Prinzip aus dem Register der erfolgten Impfungen schätzen. Die Wirksamkeit jedoch muss man kennen, bevor überhaupt die Entscheidung über das Impfprogramm fällt. Ihrer Definition entsprechend geht das nur mit Hilfe einer Kohortstudie, in der man eine Kohorte von geimpften und eine Kohorte von nicht geimpften Kindern verfolgt und sie miteinander im Laufe der Zeit vergleicht hinsichtlich der Zahl der Erkrankungen. Das ist sehr aufwendig aber unumgänglich. Zum Beispiel nahmen an der ursprünglichen Studie des ersten Impfstoffs gegen Kinderlähmung ungefähr eine halbe Million Kinder teil.

Es ist aber wichtig, die Wirksamkeit auch später, wenn das Programm schon läuft, immer wieder neu zu schätzen zur Kontrolle unter den spezifischen lokalen Bedingungen, die sich überdies noch im Lauf der Zeit ändern können. Jedes Mal solche riesigen Kohortstudien anzustellen ist unmöglich. Man kann nun folgendes zeigen. Die Wirksamkeit $h$ lässt sich darstellen als eine rationale Funktion des Durchimpfungsgrades $g$ einerseits und einer Zahl $d$ andererseits, die folgendermaßen definiert ist: sie ist der Bruchteil der geimpften Kinder unter den Fällen. Einen Schätzwert dieses Bruchteils bekommt man aus den Registern der Konsultationen mit Hilfe dessen, was man eine teilweise auf Register gestützte Fallstudie nennen würde, und zwar teilweise deshalb, weil man ja zusätzlich zu den ohnehin im Register vorhandenen Variablen noch für jeden diagnostizierten Fall den Impfstatus hinzufügen muss. Den zu finden kann zwar unter den Bedingungen eines Entwicklungslandes einige Arbeit kosten, ist aber im Prinzip möglich. Die gesamte Studie, d.h. die Schätzung von sowohl $g$ als auch $d$ und damit der Wirksamkeit $h$ läuft dann auf eine teilweise auf Register gestützte Hybridstudie hinaus. Man kann sie mit den in meinem früheren Vortrag beschriebenen Methoden auswerten.

Auf den Effekt anderer präventiver Maßnahmen wie Hygiene oder Gesundheitserziehung will ich nicht weiter eingehen. Auch darüber kann man sehr viel aus den Registern erfahren wie übrigens auch über die Arbeit der Gesundheitsstationen im Allgemeinen, worauf die Kontrolle dieser Arbeit beruht. Ich möchte stattdessen zum Abschluss noch etwas über Faktoren sagen, die die Entstehung von Krankheiten beeinflussen.

Sehen wir uns zunächst das Register der Konsultationen noch einmal an. Da stehen also einerseits, in der Spalte ,Diagnose“, die Werte der Ergebnisvariablen $X$. Andererseits 
gibt es verschiedene Vorhersagevariable $Y$ wie die Zeit, nämlich das Datum der Konsultation, den Ort, nämlich die Adresse des Patienten, das Alter, das Geschlecht und der Beruf. Wir haben schon festgestellt, dass die Indikatoren „Häufigkeit der diversen Krankheiten, aufgeschlüsselt nach Zeit, Ort, Alter, Geschlecht und Beruf“ wie sie dann z.B. in den statistischen Jahrbüchern des Gesundheitswesens erscheinen, das Resultat von routinemäßigen auf Register gestützten Querschnittsstudien sind. Vom epidemiologischen Standpunkt aus beschreiben diese Indikatoren den Einfluss oder die Wirkung der Faktoren Zeit, Ort, Alter, Geschlecht und Beruf auf das Krankheitsspektrum. Das ist aber nicht nur für die oberen Gesundheitsbehörden von Belang, sondern eigentlich viel mehr für die Gesundheitsstationen selbst. Dort kann man z.B. die geographische Verteilung der Bakterienruhr in einer Gemeinde mit hygienischen Bedingungen oder stagnierenden Gewässern in Verbindung bringen und daraus praktische Folgerungen für die Prävention ziehen. Auch die zeitliche Entwicklung von Krankheitshäufigkeiten ist oft für die Planung der Arbeit der Station nützlich. All das ist allereinfachste aber doch solide und relevante epidemiologische Forschung, die die Krankenschwestern und Krankenpfleger selbst machen können und machen sollten.

Wenn man sich nun für den Einfluss anderer Faktoren interessiert, die nicht schon im Register stehen, dann muss man sie eben für die Dauer einer Querschnittsstudie als zusätzliche Variable hinzufügen. Solche Faktoren wären z.B. die Lebensgewohnheiten oder Umwelteinflüsse. Die Auswertung solcher teilweise auf Register gestützten Studien kann schon recht tief liegende epidemiologische Methoden erfordern.

Wie steht es schließlich mit auf das Register der Konsultationen gestützten Kohortund Fall-Kontroll-Studien? Für Kohortstudien ist die Antwort einfach: sie sind unmöglich, weil man in diesem Register keine Kohorten verfolgen kann. Fall-Kontroll-Studien dagegen sind unter Umständen nicht nur möglich sondern auch sehr nützlich. Die Fälle stehen ja alle im Register. Die Schwierigkeit liegt, wie immer bei Fall-Kontroll-Studien, in der Konstituierung der Kontrollgruppe, die hier auch aus dem Register erfolgen müsste. Bei Problemen, in denen man die Population der gelegentlich zu einer Konsultation erscheinenden Personen als hinreichend repräsentativ für die Gesamtbevölkerung ansehen kann, geht es; bei anderen sollte man es lieber nicht tun.

Adresse des Autors:

Klaus Krickeberg

Großer Kamp 4

D-33619 Bielefeld

Deutschland

E-mail: krik@ideenwelt.de 